\title{
Posttranslational Regulation of Microtubule-Related Molecules as a Potential Therapeutic Target for Neurodegeneration
}

\author{
Kazuhiro Nakamura ${ }^{1}$
}

Treatments of neurodegenerative disorders such as Alzheimer's disease (AD), Huntington's disease (HD), Parkinson's disease (PD), Amyotrophic lateral sclerosis (ALS) and Spinocerebellar ataxia (SCA) have long been subjects of intense investigation by numerous research groups. I am working on neurodegeneration aiming at finding common targets to treat multiple neurodegenerative disorders. I introduce recent topics on therapeutic approach for neurodegenerative diseases in both model animals and man in this essay.

When we plan out the therapeutic strategy, an approach to reduce causative factors is theoretically straightforward. As for AD brain, the neuropathological hallmarks are extracellular lesions called senile plaques made of beta-amyloid $(A \beta)$ generated from its precursor protein APP and neurofibrillary tangles made of hyperphosphorylated tau. The mediators that underlie generation of $\mathrm{A} \beta$ and hyperphosphorylated tau have been vigorously explored. Therefore, the first attempt to reduce the causative factors is pharmacological inhibition of production of $A \beta$ and hyperphosphorylated tau. The second attempt to reduce them is to directly block pathogenic functions of $\mathrm{A} \beta$ and hyperphosphorylated tau by immunotherapy and indeed, A $\beta$ immunotherapy have entered clinical trials. Before entering clinical trials, the effectiveness of active immunization with synthetic $\mathrm{A} \beta 42$ and peripheral administration of anti-A $\beta$ antibody were assessed using AD model mice, and reduction of $\mathrm{A} \beta$ and behavioral correction was observed. However, emergence of meningoencephalitis due to inflammatory $\mathrm{T}$ cell response was seen in some patients in clinical trial of $\mathrm{A} \beta$ immunotherapy. So, efforts were made to reduce the side effect. For instance, modified protocol such as a peptide prime-DNA boost immunization protocol of $A \beta 42$ and narrowing down the region in $\mathrm{A} \beta$ sequence as the immunogen were tested and obtained absence of the A $\beta 1-42 \mathrm{~T}$ cell response in animals. Remarkable news is a phase $\mathrm{I}$, double -blind, placebo-controlled, 52-week study of 58 AD patients in Sweden reported in 2012. They designed to induce $\mathrm{N}$-terminal $\mathrm{A} \beta$-specific antibodies and got acceptable antibody response, and recorded no clinical or subclinical cases of meningoencephalitis. Although some patients reported serious adverse events, none was thought to be related to the study drug. Larger trials might confirm the availability of the antibody in near future.

Regarding the other pathogenic constituent of AD, tau immunotherapy also revealed favorable effects in animals although its progress is behind the $\mathrm{A} \beta$ immunotherapy. Tau 379-408 $\left(\mathrm{P}-\mathrm{Ser}_{396,404}\right)$, Tau 195$213\left(\mathrm{P}_{202 / 205}\right)$, Tau 207-220 $\left(\mathrm{P}_{212 / 214}\right)$ and Tau 224-238 $\left(\mathrm{P}_{231}\right)$ were used for immunotherapy of tau transgenic mice. These mice showed reduction of aggregates and behavioral improvements but were essentially free of clinical neurological deficits. As the mechanism, the possible involvement of the lysosomal system in the reduction of tangles was suggested. In case of hereditary neurodegeneration, it is better, as a matter of course, to prevent onset of symptoms. In this context, refined immunotherapy targeting hereditary neurodegeneration that starts from pre-symptomatic stage is an attractive approach.

Apart from the clearance of the causative factors as mentioned above, protection of neurons that are destined to degenerate, leaving the pathogenic factors intact, is also reasonable. That is like repair of damaged parts. Such an idea was brought from a notable observation seen in AD that deficits in the neuronal processes such as synaptic function can be found much earlier than somatic cell death. Cytoskeletons, cytosolic proteins, mitochondria, synaptic vesicle precursors and secretory products are transport-

1 Gunma University Graduate School of Medicine, 3-39-22 Showa-machi, Maebashi, Gunma 371-8511, Japan

Received: May 30, 2013

Address : KAZUHIRO NAKAMURA Gunma University Graduate School of Medicine, 3-39-22 Showa-machi, Maebashi, Gunma 371-8511, Japan 
ed along microtubule (MT) in the axons. Failure of the axonal transport likely leads to axonal degeneration and subsequent cell death. Therefore, support of the transport along neuronal processes likely ameliorate or delay somatic cell death. Indeed, the brainpenetrant MT-stabilizing agent, epothilone D improved fast axonal transport and cognition in AD model mice. In addition, abnormal MT-based transport also contributes to the neuronal toxicity in HD.

MT consists of heterodimers of two small globular $\alpha$-tubulin and $\beta$-tubulin. The mechanism by which tubulins form highly complex and functional microtubule structure depends on the interacting proteins. MT-associated proteins such as tau stabilize MT and motor proteins, kinesins and dyneins, can move on MT. Deficits in any of the molecules above can cause the transport failure. Intriguingly, both MT-associated protein tau and tubulin undergo posttranslational regulation. The posttranslational regulation of tau that is related to MT function is protein conformational change. Protein conformation of tau is spontaneously converted. However, the conversion rate is accelerated in the presence of an isomerase, ${ }^{1-3}$ and a specific tau conformation fails to stabilize MT (Fig. 1). ${ }^{1,2,4}$ On the other hand, the action of posttranslational regulation of tubulin differs from that of tau.
Tubulin is subjected to the detyrosination, polyglutamylation, polyglycylation, acetylation and phosphorylation (Fig. 1) and each type of the amino acid modification of tubulin changes MT function. Acetylation and polyglutamylation of tubulin can be regulated by histone deacetylase, cytosolic carboxypeptidase and/or tubulin tyrosine ligase-like proteins. I believe that manipulation of posttranslational regulations of MT-related molecules is worth a try in neurodegeneration model primates. Such data would be a basis of MT-targeted therapy in man in future.

\section{References}

1. Nakamura K, Zhou XZ, Lu KP. Distinct functions of cis and trans phosphorylated tau in Alzheimer's disease and their therapeutic implications. Curr Mol Med In press.

2. Nakamura K, Zhen Zhou X, Ping Lu K. Cis phosphorylated tau as the earliest detectable pathogenic conformation in Alzheimer disease, offering novel diagnostic and therapeutic strategies. Prion 2013; 7(2) : 117-120.

3. Nakamura K, Kosugi I, Lee DY, et al. Prolyl isomerase Pin1 regulates neuronal differentiation via $\beta$-catenin. Mol Cell Biol 2012; 32(15) : 2966-2978.

4. Nakamura K, Greenwood A, Binder L, et al. Proline isomer-specific antibodies reveal the early pathogenic tau conformation in Alzheimer's disease. Cell 2012; 149(1) : 232-244.

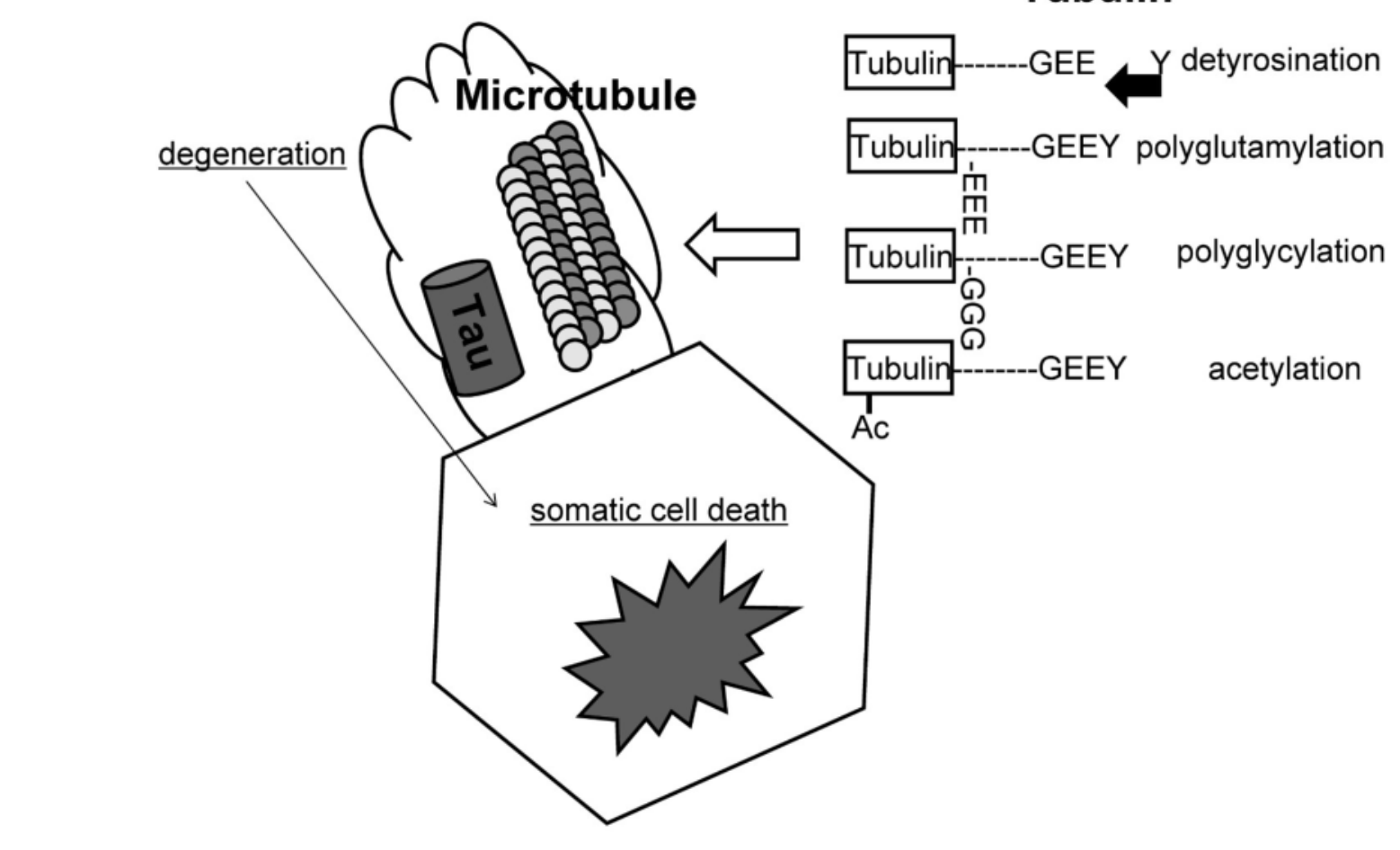

\section{Tubulin}

Fig. 1. Posttranslational regulations of MT-related molecules. Conformational change of tau and modifications of tubulins affect stability of MT, leading to degeneration of neuronal processes and probably subsequent somatic cell death. 